\title{
El gen del receptor cannabinoide tipo 2 se asocia con la comorbilidad entre esquizofrenia y dependencia de cannabis y el gen de la enzima amidohidrolasa de ácidos grasos se asocia con la dependencia de cannabis en población española
}

\author{
Cannabinoid receptor type 2 gene is associated with \\ comorbidity of schizophrenia and cannabis dependence \\ and fatty acid amide hydrolase gene is associated with \\ cannabis dependence in the Spanish population
}

Francisco Arias Horcajadas*, José Ramón Dávila Píriz**, Alba Parra González*, Sergio Sánchez Romero***, Eva Sánchez-Morla*, Israel Ampuero Sánchez****, José Antonio Ramos Atance*****.

* Servicio de Psiquiatría. Hospital Doce de Octubre. Madrid.

** Instituto de Enseñanza Secundaria (IES) Alfonso X El Sabio (Toledo).

*** Servicio de Psiquiatría. Hospital Universitario Fundación de Alcorcón. Madrid.

**** Colegio Camino Real. Torrejón de Ardoz. Madrid.

***** Departamento de Bioquímica y Biología Molecular. Facultad de Medicina. Universidad Complutense de Madrid.

\section{Resumen}

El sistema cannabinoide se ha asociado con varios trastornos psiquiátricos como la esquizofrenia y las adicciones. Diversos estudios han observado que algunos polimorfismos del receptor cannabinoide tipo 2 (CNR2), del receptor cannabinoide tipo 1 (CNR1) y del gen de la enzima amido hidrolasa de ácidos grasos $(F A A H)$ pueden ser factores de riesgo de estos trastornos. Hemos analizado diversos polimorfismos del sistema cannabinoide en pacientes diagnosticados de esquizofrenia sin trastorno por uso de sustancias $(\mathrm{n}=379)$, esquizofrenia con trastorno por uso de cannabis $(\mathrm{n}=124)$, dependientes de cannabis $\sin$ psicosis asociada $(\mathrm{n}=71)$ y un grupo de control (316) procedentes de diversos hospitales y centros de asistencia sanitaria españoles. Hemos encontrado una asociación entre los polimorfismos rs35761398 y rs12744386 del CNR2 con la presencia de esquizofrenia y trastorno por uso de cannabis comórbido y una pérdida de heterocigosidad en el polimorfismo rs324420 del gen $F A A H$ con la dependencia de cannabis en población española. Los polimorfismos rs35761398 y rs12744386 en CNR2 son factores de riesgo para esquizofrenia en sujetos dependientes de cannabis. La pérdida de heterocigosidad en el polimorfismo rs324420 en el gen FAAH es un factor de riesgo para la dependencia de cannabis.

Palabras clave: Trastorno por uso de cannabis; esquizofrenia; polimorfismos; gen del receptor cannabinoide tipo 2; gen del receptor cannabinoide tipo 1; gen de la enzima amido hidrolasa de ácidos grasos.

\begin{abstract}
The endocannabinoid system has been associated with various psychiatric disorders, such as schizophrenia or addictive disorders. Recent studies have found that some polymorphisms in the cannabinoid receptor type 2 (CNR2), cannabinoid receptor type 1 (CNR1) and fatty acid amide hydrolase $(F A A H)$ genes could play an important role as risk factors in the etiology of these diseases. We analysed different cannabinoid gene polimorphisms from non-substance using patients diagnosed with schizophrenia $(\mathrm{n}=379)$, schizophrenic patients with cannabis use disorders $(n=124)$, cannabis users who did not have psychoses $(n=71)$, and 316 controls from various Spanish hospitals and health centres. We found a statistical association between polymorphisms rs35761398 and rs12744386 in the CNR2 gene and comorbidity of schizophrenia and cannabis dependence, as well as an association between loss of heterozygosity (overdominance) for polymorphism rs324420 in the $F A A H$ gene and cannabis dependence in a Spanish population sample. The rs35761398 and rs12744386 polymorphisms in the CNR2 gene are genetic risk factors for schizophrenia in cannabis-dependent subjects. Loss of heterozygosity for polymorphism rs324420 in the FAAH gene is a genetic risk factor for cannabis dependence in this population. Keywords: Cannabis use disorder; schizophrenia; polymorphisms; cannabinoid receptor type 2 gene; cannabinoid receptor type 1 gene; fatty acid amide hydrolase gene.
\end{abstract}


L

a esquizofrenia es un trastorno mental grave con una prevalencia mundial entre $0,5 \%$ y $1,0 \%$ y tiene un enorme impacto social y económico (Andreasen, 1995; Dong et al., 2019). Diferentes estudios epidemiológicos han sugerido que el cannabis podría ser un factor de riesgo para el desarrollo de la esquizofrenia (Marconi, Di Forti, Lewis, Murray y Vassos, 2016). Además, los conocidos efectos psicotrópicos de los cannabinoides y la distribución de los receptores cannabinoides en el cerebro sugieren que el sistema endocannabinoide puede estar involucrado en la esquizofrenia (Fakhoury, 2017; Minichino et al., 2019) y en los trastornos adictivos (Manzanares et al., 2018; Van Hell et al., 2012). Un estudio identificó una asociación entre el consumo temprano de cannabis, un menor grosor cortical y un alto riesgo poligénico de psicosis en adolescentes. Este hallazgo implica procesos que subyacen a la maduración cortical en la mediación de la relación entre el consumo de cannabis y la propensión a la esquizofrenia (French et al., 2015), lo que indica que el cannabis podría desempeñar un papel en el desarrollo de la psicosis mediante la alteración de los circuitos neuronales en sujetos con vulnerabilidad genética (Aas et al., 2017; Fonseca-Pedrero, Lucas-Molina, Pérez-Albéniz, Inchausti y Ortuño-Sierra, 2019; French et al., 2015; García-Álvarez, Gomar, García-Portilla y Bobes, 2019; Parkar et al., 2011).

Los genes CNR1, CNR2 y FAAH codifican algunas de las proteínas asociadas con el sistema endocannabinoide. Los receptores $\mathrm{CB} 1$ se localizan principalmente en el sistema nervioso central y son abundantes en los ganglios basales, hipocampo, cerebelo y áreas corticales (Herkenham et al., 1991). Los receptores CB2 se encontraron inicialmente en el sistema inmunológico (Galiègue et al., 1995); sin embargo, también se ha demostrado su presencia en neuronas y células gliales de múltiples áreas cerebrales (corteza cerebral, hipocampo, amígdala, estriado, tálamo, cerebelo, etc.) (Gong et al., 2006; Onaivi et al., 2006). La enzima amido hidrolasa de ácidos grasos (FAAH) es responsable de la hidrólisis de la anandamida, un ligando endógeno de este sistema (Deutsch, Ueda y Yamamoto, 2002).

Algunos estudios han sugerido una asociación entre el gen CNR1 (que codifica el receptor CB1) y la incidencia de esquizofrenia (Chavarría-Siles et al., 2008; Leroy et al., 2001; Martínez-Gras et al., 2006; Ujike et al., 2002) y trastornos por uso de sustancias, como el trastorno por consumo de cannabis (Gerra et al., 2018; Hartman et al., 2009). Sin embargo, la evidencia sigue siendo heterogénea y controvertida para ambos resultados. Gouvêa et al. (2017) analizaron sistemáticamente todos los ensayos existentes sobre las variantes genéticas del $C N R 1$ y esquizofrenia y destacaron la alta heterogeneidad de los resultados. Un polimorfismo que consta de nueve alelos que contienen secuencias repetidas de $(\mathrm{AAT})_{7-15}$ se ha utilizado en estudios de asociación sobre el gen $C N R 1$ y enfermedades mentales y abu- so de sustancias en poblaciones diferentes, con resultados contradictorios (Ballon et al., 2006; Chavarría-Siles et al., 2008; Comings et al., 1997; Martínez-Gras et al., 2006; Tsai, Wang y Hong, 2000; Ujike et al., 2002).

En los últimos años, el receptor CB2 ha sido el foco de atención debido a su función como modulador de la neuroinflamación (Javed, Azimullah, Haque y Ojha, 2016; Kong, Li, Tuma y Ganea, 2014; Malfitano, Basu, Maresz, Bifulco y Dittel, 2014), los procesos de memoria (García-Gutiérrez et al., 2013), y el procesamiento de recompensas, y por su papel en la drogadicción y psicosis (Onaivi, Ishiguro, Gu y Liu, 2012; Xi et al., 2011). Se halló que la frecuencia del alelo CC de rs35761398 (variante R63), el alelo C de rs12744386, el haplotipo del alelo CC de rs35761398 y el alelo C de rs12744386 (CC/C) estaba aumentada de manera significativa en una muestra de japoneses con esquizofrenia en comparación con sujetos de control (Ishiguro et al., 2010). Se observó una respuesta significativamente menor a los ligandos CB2 en células cultivadas transfectadas con el alelo CC de rs35761398 en comparación con aquellas transfectadas con el alelo TT, y se encontraron niveles de proteína y ARNm del receptor CB2 significativamente más bajos en el cerebro humano con genotipos $\mathrm{C} / \mathrm{C}$ y C/T de rs12744386 en comparación con los genotipos T/T (Ishiguro et al., 2010).

Por otro lado, un polimorfismo de un solo nucleótido (SNP) común rs324420 (C385A) en el gen FAAH humano se ha relacionado con el abuso de sustancias, por ejemplo, cannabis (Tyndale, Payne, Gerber y Sipe, 2007), cocaína (Patel et al., 2018) y metanfetamina (Zhang, Liu, Deng, Ma y Liu, 2020).

El objetivo de este estudio fue investigar la asociación genética entre el haplotipo (AAT) ${ }_{7-15}$ del gen CNR1, el polimorfismo rs324420 del $F A A H$, los polimorfismos rs35761398 y rs12744386 del gen $C N R 2$, y la esquizofrenia y la dependencia de cannabis en una muestra de sujetos españoles.

\section{Métodos}

\section{Participantes}

En este estudio, se analizaron 379 pacientes con esquizofrenia, 124 pacientes con esquizofrenia y trastorno por consumo de cannabis (CUD) (grupo dual), 71 sujetos con CUD sin psicosis (grupo de cannabis) y 316 controles que no estaban relacionados entre sí. Los diagnósticos se realizaron mediante entrevista clínica conforme al DSM-IVTR. Los pacientes (ambulatorios y hospitalizados) fueron reclutados en diferentes hospitales de la Comunidad de Madrid y Castilla-La Mancha. Los consumidores de cannabis sin psicosis fueron reclutados de centros de adicciones o asociaciones de usuarios en la Comunidad de Madrid. Los criterios de inclusión fueron: edad mínima de 18 años, origen étnico español y caucásico, y firma del con- 
sentimiento informado. Los criterios de exclusión fueron: ser o tener familiares de primer grado de otro origen étnico, de países distintos de España, presencia de trastornos mentales distintos a los del objeto de estudio, dependencia de drogas distintas del cannabis o tabaco, patología cerebral orgánica comórbida u otra condición médica grave y negarse a participar o firmar el consentimiento informado. Se excluyeron a los pacientes con un diagnóstico de dependencia de drogas distinta del cannabis o el tabaco, aunque se incluyeron si eran usuarios de drogas pero sin dependencia.

La población de control consistió en 316 voluntarios individuales que no estaban relacionados entre sí y fueron reclutados de la población española. Eran personal sanitario y administrativo de los centros de salud atendidos por los pacientes y acompañantes de los pacientes. Todos participaron en una entrevista clínica para excluir otras patologías psiquiátricas.

\section{Instrumentos de evaluación}

Mediante entrevista clínica se obtuvieron variables sociodemográficas, antecedentes personales y familiares y datos relacionados con el consumo de sustancias. Además, en esa primera entrevista se evaluó la sintomatología psicótica mediante la Escala de síndrome positivo y negativo de la esquizofrenia (PANSS).

Escala PANSS (Escala de síndrome positivo y negativo). La Escala de síndrome positivo y negativo, desarrollada por Kay, Fiszbein y Opler (1987), cuya versión en español fue creada por Peralta y Cuesta (1994), es una de las herramientas más utilizadas para evaluar los síntomas en pacientes con esquizofrenia. Es una escala administrada por médicos que se completa durante una entrevista semiestructurada de aproximadamente 45 minutos de duración. En su versión original, la PANSS consta de 30 ítems agrupados en tres factores: síndrome positivo (7 ítems), síndrome negativo (7 ítems) y psicopatología general (16 ítems). En este estudio, además de utilizar la puntuación total de la PANSS, también se utilizaron las tres subescalas (psicopatología positiva, negativa y general).

\section{Procedimiento del estudio}

Pacientes hospitalizados y ambulatorios atendidos en diferentes centros de salud mental (Hospital Universitario Fundación Alcorcón (Madrid), Hospital Ramón y Cajal (Madrid), Hospital Virgen de la Luz (Cuenca), Hospital Universitario de Guadalajara, Clínica Nuestra Señora de La Paz (Madrid)), que cumplieron los criterios de inclusión y exclusión y estaban dispuestos a participar en nuestro estudio, fueron reclutados prospectivamente y firmaron un consentimiento informado. Se reclutaron sujetos con dependencia de cannabis de diferentes centros de tratamiento de drogodependencias de la Comunidad de Madrid (Majadahonda, Alcorcón, Arganzuela, Vallecas, Latina).

Un total de 27 sujetos fueron excluidos ya sea porque se negaron a participar en el estudio o firmar el consentimiento informado.

\section{Extracción de ADN y genotipado}

Se obtuvo ADN de leucocitos presentes en muestras de sangre periférica anticoagulada con EDTA, utilizando el Método Sambrook y el kit DNeasy Blood y Tissue (Qiagen).

Después de la extracción de ADN de sangre periférica, se analizaron los diferentes polimorfismos mediante métodos basados en PCR. La genotipificación del polimorfismo rs324420 en el gen $F A A H$ se realizó mediante el método SSCP (polimorfismo de conformación de cadena simple) (GeneGel Excel 12.5/24 Kit, GE Healthcare) (ver la Tabla 1 para las secuencias de los cebadores). La genotipifica-

Tabla 1. Cebadores y técnicas utilizadas para el análisis de muestras.

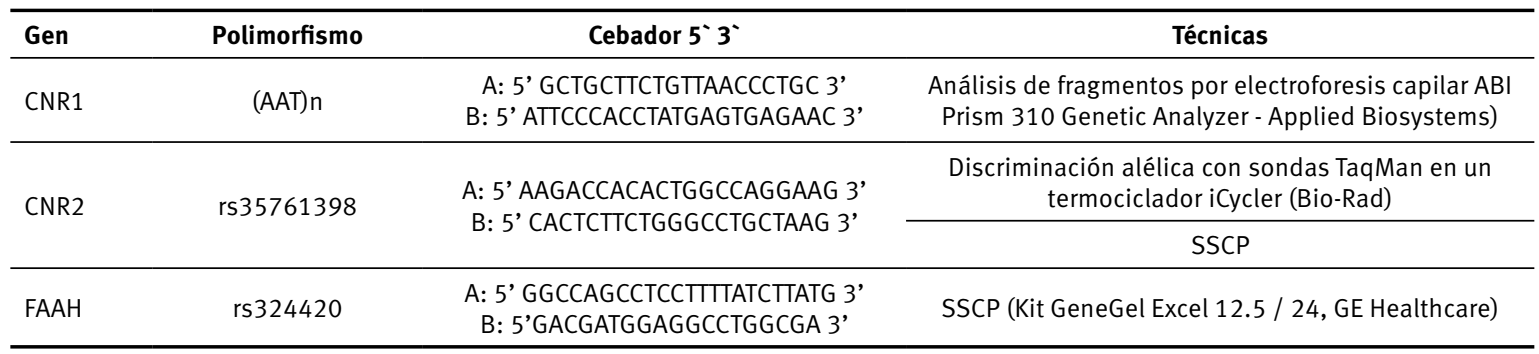

Nota. Polimorfismo de Conformación de Cadena Simple (SSCP).

ción del polimorfismo (AAT) ${ }_{7-15}$ 3'UTR se realizó utilizando una técnica de análisis de fragmentos mediante electroforesis capilar (ABI Prism 310 Genetic Analyzer - Applied Biosystems) (ver la Tabla 1 para las secuencias de los cebadores). Se utilizó el estándar GeneScan-500 LIZ como marcador de tamaño (Applied Biosystems). Los resultados se analizaron con el software GeneMapper 4.0. La genotipificación de los polimorfismos rs35761398 y rs12744386 en el gen CNR2 se realizó usando discriminación alélica con sondas TaqMan en un termociclador iCycler (Bio-Rad) (ver la Tabla 1 para las secuencias de sonda TaqMan y de los cebadores). 


\section{Cuestiones éticas}

La participación en el estudio fue voluntaria y todos los participantes dieron su consentimiento por escrito para participar. El estudio fue aprobado por el Comité Ético de Investigación Clínica del Hospital Universitario Fundación Alcorcón (Madrid).

\section{Análisis estadístico}

La distribución de genotipos se comparó con el valor predecible del equilibrio de Hardy-Weinberg. Los grupos de control y de casos cumplían el equilibrio de Hardy-Weinberg respecto de las frecuencias de alelos y genotipo para los polimorfismos estudiados (Tabla 2).

Tabla 2. Equilibrio de Hardy-Weinberg.

\begin{tabular}{lcccc}
\hline GEN & Polimorfismo & Sexo & $\chi^{2}$ & Valor $\mathbf{p}$ \\
\hline CNR1 & \multirow{2}{*}{ (AAT) $n$} & Mujeres &, 5389 &, 7638 \\
& & Hombres & 3,2414 &, 1978 \\
\hline \multirow{2}{*}{ CNR2 } & \multirow{2}{*}{ rs35761398 } & Mujeres &, 0467 &, 9769 \\
& & Hombres &, 1874 &, 9106 \\
\hline \multirow{2}{*}{ FAAH } & \multirow{2}{*}{ rs324420 } & Mujeres & 1,5451 &, 4183 \\
& & Hombres &, 1229 &, 9404 \\
\hline
\end{tabular}

Nota. Dos grados de libertad excepto rs6323 y rs1799836 en el hombre (hemicigosidad), calculado para 1 grado de libertad.

Los nueve alelos que contienen secuencias repetidas de $(\mathrm{AAT})_{7-15}$ se distribuyeron de acuerdo con el manuscrito de Comings et al. (1997) en un grupo de alelos cortos con menos de 11 repeticiones de tripletes AAT (genotipo $<5)$ y otro grupo de alelos largos $>11$ repeticiones (genotipo $>5$ ). Así, los pacientes y controles se subdividieron en tres grupos por genotipo: individuos con $<5 /<5,>5 />5$ y $<5 />5$. Estos datos se utilizaron como variables cualitativas. La hipótesis de una asociación entre genotipos y grupos se probó mediante la prueba de chi-cuadrado de Pearson y donde los tamaños de las células eran iguales o menores a 5 mediante la prueba exacta de Fisher. Se aplicó la corrección de Bonferroni.

Se utilizó una prueba de valor $p$ bilateral y los valores $p$ $<, 05$ se consideraron estadísticamente significativos. El análisis se llevó a cabo utilizando el software en línea OpenEpi (Open Source Epidemiologic Statistics for Public Health).

\section{Resultados}

Las tablas 3 y 4 resumen los datos sociodemográficos y clínicos. No se observaron diferencias estadísticamente significativas en ninguna de las variantes polimórficas estudiadas en el análisis poblacional en función de variables como sexo o edad al inicio del consumo de cannabis o síntomas psicóticos.

\section{CNR1}

Respecto del polimorfismo (AAT) ${ }_{7-15}$ 3'UTR en el gen CNR1, no hallamos diferencias estadísticamente significativas entre el grupo de pacientes con esquizofrenia, grupos dual y cannabis y la población de control al analizar las frecuencias de alelos y genotipos (Tabla 5).

\section{FAAH}

En cuanto al polimorfismo rs324420 en el gen $F A A H$, no hallamos diferencias estadísticamente significativas entre la población de control y ambos sujetos con esquizofrenia y esquizofrenia comórbida y dependencia de cannabis al comparar las frecuencias de alelos y genotipos (Tabla 5). Asumiendo un modelo de sobredominancia, se encontraron diferencias estadísticamente significativas entre los sujetos con dependencia de cannabis y el grupo de control (Tabla 6).

\section{CNR2}

En cuanto al análisis del polimorfismo rs35761398 en el gen $C N R 2$, al comparar el grupo dual con los grupos

Tabla 3. Descripción general de la muestra.

\begin{tabular}{|c|c|c|c|c|}
\hline & $\begin{array}{l}\text { Controles } \\
N=316\end{array}$ & $\begin{array}{l}\text { Esquizofrenia } \\
\qquad N=379\end{array}$ & $\begin{array}{c}\text { Esquizofrenia }+ \\
\text { dependencia de cannabis } \\
N=124\end{array}$ & $\begin{array}{l}\text { Dependencia de cannabis } \\
\qquad N=71\end{array}$ \\
\hline Hombre (\%) & 42,72 & 60,16 & 87,90 & 70,42 \\
\hline $\begin{array}{l}\text { Edad media en el momento de la } \\
\text { prueba (p25-p75) }\end{array}$ & $31(28-37)$ & $37(31-50)$ & $29(26-36)$ & $28(25-34)$ \\
\hline Edad media al diagnóstico (p25-p75) & & $25(20-32)$ & $25(20-28)$ & $23(19-30)$ \\
\hline $\begin{array}{l}\text { Edad media del primer consumo de } \\
\text { cannabis (p25-p75) }\end{array}$ & & & $16(15-18)$ & $16(15-17)$ \\
\hline \multicolumn{5}{|l|}{ Puntuación PANSS: media (IC del $95 \%$ ) } \\
\hline Escala Positiva & & $22,6(20,7-24,5)$ & $21,5(18,8-24,1)$ & \\
\hline Escala Negativa & & $23,8(22,0-25,6)$ & $20,1(17,0-23,2)$ & \\
\hline Escala Global & & $36,3(34,2-38,3)$ & $33,5(31,0-35,9)$ & \\
\hline
\end{tabular}

Nota. PANSS: Escala de síndrome positivo y negativo de la esquizofrenia. $\mathrm{P}=$ percentil. $\mathrm{IC}=$ intervalo de confianza. Predominio masculino en esquizofrenia + depen dencia de cannabis vs. esquizofrenia (chi ${ }^{2}=32,53$, p <,001, OR: 4,81, IC: $\left.95 \%: 2,70-8,58\right)$. 4,81, IC: $95 \%: 2,70-8,58$ ). 
Francisco Arias Horcajadas, José Ramón Dávila Píriz, Alba Parra González, Sergio Sánchez Romero,

Eva Sánchez-Morla, Israel Ampuero Sánchez, José Antonio Ramos Atance

Tabla 4. Porcentajes de consumo de drogas entre grupos.

\begin{tabular}{lcccc}
\hline & $\begin{array}{c}\text { Esquizofrenia y } \\
\text { dependencia de cannabis } \\
\mathbf{N = 1 2 4}\end{array}$ & $\begin{array}{c}\text { Dependencia de } \\
\text { cannabis } \\
\mathbf{N = 7 1}\end{array}$ & $\begin{array}{c}\text { Esquizofrenia } \\
\mathbf{N = 3 7 9}\end{array}$ & $\begin{array}{c}\text { Controles } \\
\mathbf{N = 3 1 6}\end{array}$ \\
\hline Cannabis (cigarrillo/día): media; mediana & 7,$5 ; 6$ & $6 ; 6$ & 0 & 0 \\
Tabaco (\%) & 92,1 & 64,3 & 48,4 & 36,8 \\
Alcohol (\%) & 48,0 & 21,4 & - & - \\
Cocaína (\%) & 32,7 & 10,7 & - & - \\
Opioides (\%) & 9,3 & 0,0 & - & - \\
Anfetaminas (\%) & 1,3 & 0,0 & - & - \\
Otros (\%) & 12,0 & 0,0 & - & - \\
\hline
\end{tabular}

Nota. Porcentaje de consumo de alcohol, cocaína, opioides, anfetaminas u otras drogas sin criterios de dependencia. En el subgrupo de esquizofrenia + dependencia de cannabis, el $52 \%$ de los sujetos solo consumía tabaco y cannabis.

Tabla 5. Distribución de frecuencias de genotipos y alelos entre subgrupos.

\begin{tabular}{|c|c|c|c|c|c|c|}
\hline $\begin{array}{l}\text { Polimorfismo } \\
\text { (gen) }\end{array}$ & \multicolumn{2}{|c|}{ Genotipo/Alelo } & $\begin{array}{c}\text { Controles } \\
\text { N (\%) }\end{array}$ & $\begin{array}{c}\text { Esquizofrenia } \\
\text { N (\%) }\end{array}$ & $\begin{array}{c}\text { Esquizofrenia }+ \\
\text { dependencia de cannabis } \\
\mathbf{N}(\%)\end{array}$ & $\begin{array}{c}\text { Dependencia de } \\
\text { cannabis } \\
\text { N (\%) }\end{array}$ \\
\hline \multirow{5}{*}{ (AAT)n (CNR1) } & \multirow{3}{*}{ Genotipo } & LL & $171(54,11)$ & $190(50,13)$ & $58(46,77)$ & $33(57,89)$ \\
\hline & & LS & $116(36,71)$ & $145(38,26)$ & $52(41,94)$ & $20(35,09)$ \\
\hline & & SS & $29(9,18)$ & $44(11,61)$ & $14(11,29)$ & $4(7,02)$ \\
\hline & \multirow{2}{*}{ Alelo } & $\mathrm{L}$ & $458(72,47)$ & $525(69,26)$ & $168(67,74)$ & $86(75,44)$ \\
\hline & & $S$ & $174(27,53)$ & $233(30,74)$ & $80(32,26)$ & $28(24,56)$ \\
\hline \multirow{5}{*}{ rs35761398 (CNR2) } & \multirow{3}{*}{ Genotipo } & $\mathrm{CC} / \mathrm{CC}$ & $101(31,96)$ & $116(30,61)$ & $24(19,35)$ & $21(29,58)$ \\
\hline & & $\mathrm{CC} / \mathrm{TT}$ & $152(48,10)$ & $181(47,76)$ & $70(56,45)$ & $34(47,89)$ \\
\hline & & $\Pi / \Pi$ & $63(19,94)$ & $82(21,64)$ & $30(24,19)$ & $16(22,54)$ \\
\hline & \multirow{2}{*}{ Alelo } & $\mathrm{CC}$ & $354(56,01)$ & $413(54,49)$ & $118(47,58)$ & $76(53,52)$ \\
\hline & & $\pi$ & $278(43,99)$ & $345(45,51)$ & $130(52,42)$ & $66(46,48)$ \\
\hline \multirow{5}{*}{ rs324420 (FAAH) } & \multirow{3}{*}{ Genotipo } & $\mathrm{CC}$ & $202(63,92)$ & $254(67,02)$ & $90(72,58)$ & $53(75,71)$ \\
\hline & & CA & $104(32,91)$ & $107(28,23)$ & $31(25,00)$ & $11(15,71)$ \\
\hline & & AA & $10(3,16)$ & $18(4,75)$ & $3(2,42)$ & $6(8,57)$ \\
\hline & \multirow{2}{*}{ Alelo } & C & $508(80,38)$ & $615(81,13)$ & $211(85,08)$ & $117(83,57)$ \\
\hline & & A & $124(19,62)$ & $143(18,87)$ & $37(14,92)$ & $23(16,43)$ \\
\hline
\end{tabular}

Nota. $\mathrm{L}=$ alelo largo. $\mathrm{S}=$ alelo corto.

Tabla 6. Resultados de asociación para el contraste de frecuencia entre el grupo con dependencia de cannabis y controles/esquizofrenia + grupos de dependencia de cannabis.

\begin{tabular}{|c|c|c|c|c|c|c|c|}
\hline & & Polimorfismo & $\begin{array}{l}\text { Procedimiento de } \\
\text { ajuste de modelo }\end{array}$ & $\chi^{2}$ & D.F. & Valor $\mathbf{p}$ & $\begin{array}{c}\text { OR } \\
\text { (IC } 95 \%)\end{array}$ \\
\hline \multirow{13}{*}{$\begin{array}{l}\text { Dependencia } \\
\text { de cannabis }\end{array}$} & \multirow{6}{*}{$\begin{array}{l}\text { Esquizofrenia }+ \\
\text { dependencia de cannabis }\end{array}$} & \multirow{3}{*}{ rs35761398 (CNR2) } & Codominancia & 2,0365 & 2 &, 3612 & \\
\hline & & & Alelos & ,9904 & 1 & ,3196 & $1,23[, 82 ; 1,86]$ \\
\hline & & & Dominante & 2,0075 & 1 & , 1565 & $1,63[, 83 ; 3,23]$ \\
\hline & & \multirow{3}{*}{ rs324420 (FAAH) } & Codominante & 5,4918 & 2 &, 0642 & \\
\hline & & & Alelos & ,1559 & 1 & ,6930 &, $89[, 51 ; 1,57]$ \\
\hline & & & Heterocigosis & 2,2743 & 1 & ,1315 &, $56[, 26 ; 1,20]$ \\
\hline & \multirow{7}{*}{ Controles } & \multirow[t]{2}{*}{ (AAT)n (CNR1) } & Codominante & ,3618 & 2 &, 8345 & \\
\hline & & & Alelos & ,3177 & 1 &, 5730 &, $88[, 56 ; 1,38]$ \\
\hline & & \multirow{2}{*}{ rs35761398 (CNR2) } & Codominante & ,4757 & 2 & ,7883 & \\
\hline & & & Alelos & ,4793 & 1 & ,4887 & $1,14[, 79 ; 1,64]$ \\
\hline & & \multirow{3}{*}{ rs324420 (FAAH) } & Codominante & 1,9360 & 2 &, 0042 & $2,74[1,45 ; 5,31]$ \\
\hline & & & Alelos & ,7574 & 1 & ,3842 & ,81 [,49; 1,31] \\
\hline & & & Heterocigosis & 8,1024 & 1 & ,0044 & $2,63[1,33 ; 5,22]$ \\
\hline
\end{tabular}


El gen del receptor cannabinoide tipo 2 se asocia con la comorbilidad entre esquizofrenia y dependencia de cannabis y el gen de la enzima amidohidrolasa de ácidos grasos se asocia con la dependencia de cannabis en población española

Tabla 7. Resultados de asociación para el contraste de frecuencia en rs35761398 entre el grupo dual y los grupos de control/esquizofrenia.

\begin{tabular}{|c|c|c|c|c|c|c|c|}
\hline Gen & Polimorfismo & Modelo & & Grupos & $\chi^{2}$ & Valor $p$ & OR (IC $95 \%)$ \\
\hline \multirow{2}{*}{ CNR2 } & \multirow{2}{*}{ rs35761398 } & \multirow{2}{*}{ Dominante $(\mathrm{CC} / \Pi+\Pi \mathrm{T} / \Pi)$} & \multirow{2}{*}{ Dual } & Controles & 6,9595 &, 0083 & $1,96[1,18 ; 3,24]$ \\
\hline & & & & Esquizofrenia & 5,8892 & ,0152 & $1,84[1,12 ; 3,02]$ \\
\hline
\end{tabular}

Tabla 8. Análisis de asociación entre genes CNR2 y FAAH.

\begin{tabular}{lcccccc}
\hline $\begin{array}{l}\text { Genotipo } \\
\text { rs35761398 - rs324420 }\end{array}$ & $\begin{array}{c}\text { Controles (a) } \\
\mathbf{N}(\%)\end{array}$ & $\begin{array}{c}\text { Esquizofrenia } \\
\text { dependencia de cannabis (b) } \\
\mathbf{N}(\%)\end{array}$ & $\begin{array}{c}\text { Ratio } \\
\mathbf{( \% a / \% ~ b ) ~}\end{array}$ & $\chi^{\mathbf{2}}$ & Valor p & OR (IC 95 \%) \\
\hline CC/CC - CC & $51(16,14)$ & $16(12,90)$ &, 7995 &, 7224 &, 3953 &, $77[, 42 ; 1,41]$ \\
CC/CC - portador A & $50(15,82)$ & $8(6,45)$ &, 4077 & 6,8337 &, $0089(1)$ &, $37[, 17 ;, 80]$ \\
Portador TT - CC & $151(47,78)$ & $74(59,68)$ & 1,2489 & 5,0407 &, 0248 & $1,62[1,06 ; 2,46]$ \\
Portador TT - Portador A & $64(20,25)$ & $26(20,97)$ & 1,0353 &, 0279 &, 8672 & $1,04[, 63 ; 1,74]$ \\
\hline & & & & 9.031 &, $0289\left(^{*}\right)$ & \\
\hline
\end{tabular}

Nota. (1) La corrección de Bonferroni implica que los valores significativos son aquellos con valores p por debajo de $, 0125 .\left(^{\star}\right) \times \div 2=9,031 ; \mathrm{GL}=3 ; \mathrm{p}=, 0289$.

de esquizofrenia y de control, se encontraron desviaciones estadísticamente significativas en las frecuencias genotípicas (Tabla 7). Suponiendo un modelo dominante para el alelo menos frecuente (TT), las diferencias en la presencia de TT eran estadísticamente significativas al comparar el grupo dual con los controles. Tanto en los consumidores de cannabis como en los controles, no se encontraron diferencias estadísticamente significativas en las frecuencias de alelos y genotipos entre los polimorfismos rs35761398 y rs12744386. Hubo una interacción entre el polimorfismo rs35761398 en el gen CNR2 y el polimorfismo rs324420 en el gen $F A A H$ (Tabla 8).

La literatura sobre el polimorfismo rs35761398 sitúa su origen en el desequilibrio de ligamiento dentro de este gen. La implicación de ligamiento funcional de estos polimorfismos en la acción del receptor y las descripciones de haplotipos infrecuentes en otras poblaciones fueron la razón para genotipar este segundo polimorfismo en el gen CNR2, con el fin de determinar si esos haplotipos estaban presentes en la muestra de este estudio. Fueron genotipados tanto en el grupo de control como en los pacientes con diagnóstico dual. Hubo un vínculo del $100 \%$ de haplotipos entre los polimorfismos rs12744386 y rs35761398 en la población estudiada, con la presencia de los siguientes haplotipos: C - CC y T - TT. Haplotipos alternativos, es decir, T - CG y C - TT, no fueron encontrados en la población incluida en este estudio.

\section{Discusión}

Diferentes estudios epidemiológicos han sugerido que el consumo de cannabis podría ser un factor de riesgo para el desarrollo de la esquizofrenia (Marconi et al., 2016). Sin embargo, solo una pequeña proporción de consumidores de cannabis desarrollan psicosis, que puede explicarse en parte por factores genéticos. La evidencia convergente de estudios en animales y humanos sugiere que el sistema endocannabinoide (ECS) está involucrado en la fisiopatología de la psicosis (Fakhoury, 2017; Minichino et al., 2019; Rodríguez-Muñoz, Sánchez-Blázquez, Callado, Meana y Garzón-Niño, 2017). Por tanto, los genes candidatos lógicos que podrían influir en la probabilidad de desarrollar psicosis incluyen CNR1, CNR2 y FAAH.

\section{CNR1}

No encontramos evidencia de asociación entre el microsatélite $C N R 1$ y la esquizofrenia, lo que es coherente con los hallazgos de otros estudios (Ballon et al., 2006; Dawson, 1995; Seifert, Ossege, Emrich, Schneider y Stuhrmann, 2007; Tsai et al., 2000). Se ha encontrado una asociación entre el subtipo hebefrénico de esquizofrenia y el polimorfismo de repetición AAT en una población japonesa y en un estudio de asociación basado en familias en una población costarricense (Chavarría-Siles et al., 2008; Ujike et al., 2002). Nuestra muestra de sujetos con esquizofrenia incluyó principalmente a pacientes de tipo paranoide (datos no mostrados), ya que la esquizofrenia hebefrénica es muy infrecuente en la población española. Los estudios de Ujike y Chavarría-Siles no encontraron diferencias significativas en la frecuencia de genotipo o de alelos entre los sujetos con esquizofrenia paranoide y los controles.

Aunque no se ha hallado una asociación entre la esquizofrenia y otros polimorfismos diferentes de CNR1 (Leroy et al., 2001; Zammit et al., 2007), otro grupo español (Martínez-Gras et al., 2006) encontró diferencias significativas para este polimorfismo entre 113 pacientes y 111 controles sanos. El alelo 4 fue más frecuente en los controles, lo que sugiere un efecto protector contra el desarrollo de la esquizofrenia. La muestra era más heterogénea e incluyó el abuso de sustancias comórbido en el grupo de personas con 
esquizofrenia, lo que podría explicar las discrepancias con nuestros hallazgos. Además, la frecuencia del alelo repetido (AAT) 12 se incrementó en sujetos con esquizofrenia con dependencia de cocaína en una población afro-caribeña (Ballon et al., 2006).

Aunque no encontramos una asociación entre este polimorfismo y la esquizofrenia, muchos otros datos sugieren que los receptores $\mathrm{CB} 1$ podrían jugar un papel clave en su patogénesis, o que podría estar relacionado con algunos de los fenotipos relacionados con esta enfermedad. En este sentido, se ha descrito una asociación entre algunos de los polimorfismos del gen CNR1 y los efectos psicomiméticos del cannabis en una población sana (Krebs, Morvan, Jay, Gaillard y Kebir, 2014), la función cognitiva de los primeros episodios psicóticos (Rojnic et al., 2019) y la respuesta farmacogenética en la psicosis (Hamdani et al., 2008). Además, se han descrito cambios en la expresión génica de CNR1 (Tao et al., 2020) y en la metilación del ADN del gen CRN1 en la esquizofrenia (D'Addario et al., 2017), así como la disponibilidad reducida de los receptores CB1 en diferentes áreas del cerebro en los primeros episodios psicóticos (Borgan et al., 2019).

Comings et al. (1997) encontraron que este polimorfismo tuvo una asociación significativa con varios tipos diferentes de drogodependencia y uso de drogas intravenosas. De acuerdo con nuestros hallazgos, otros autores no encontraron una asociación entre el polimorfismo AAT y el abuso de sustancias (Covault, Gelernter y Kranzler, 2001; Heller, Schneider, Seifert, Cimander y Stuhrmann, 2001; Li et al., 2000). La comparación de distribuciones de alelos entre diferentes grupos étnicos mostró una marcada variación genética entre poblaciones (Comings et al., 1997; Li et al., 2000; Ujike et al., 2002). Es importante señalar que, en nuestra muestra, todos los pacientes eran caucásicos.

\section{FAAH}

$\mathrm{Al}$ comparar pacientes con esquizofrenia con o sin trastorno por consumo de cannabis con los controles, no se encontraron diferencias significativas con respecto a las frecuencias alélicas o la distribución del genotipo del gen $F A A H$. Esto es coherente con los hallazgos de Morita et al. (2005) en relación con una población japonesa, y los resultados recientemente publicados de Hindocha et al. (2020), que tampoco encontraron una asociación significativa entre el genotipo rs324420 y las experiencias psicóticas en los consumidores de cannabis. Bioque et al. (2019) analizaron los genotipos de 321 pacientes con un primer episodio de psicosis. Se analizaron un total de 15 SNP de CNR1, CNR2 y $F A A H$, pero solo encontraron significación estadística en el caso del polimorfismo rs2295633 del gen $F A A H$. Los portadores homocigotos del alelo $\mathrm{T}$ que eran consumidores de cannabis tenían una mayor probabilidad de presentar un episodio psicótico que los consumidores de cannabis sin este genotipo. No se halló suficiente significación esta- dística con respecto al polimorfismo rs324420. Watts et al. (2020) encontraron recientemente que niveles más bajos de FAAH se asociaron con síntomas psicóticos más graves. Estos resultados fueron independientes de la exposición al cannabis.

Encontramos una asociación entre el polimorfismo rs324420 en el gen $F A A H$ y la dependencia de cannabis. La presencia de un menor número de heterocigotos en el polimorfismo rs324420 en el gen $F A A H$ se asoció con la dependencia de cannabis, que nos lleva a hipotetizar que, de acuerdo con un modelo de sobredominancia, el genotipo heterocigótico confiere cierta protección contra esta dependencia. Por tanto, la heterocigosidad podría ser un equilibrio entre las demandas de flexibilidad y estabilidad en las vías neuronales implicadas. Al igual que en nuestra investigación, otros autores han encontrado una asociación entre los homocigotos AA o CC de este polimorfismo y los trastornos por uso de sustancias (Flanagan, Gerber, Cadet, Beutler y Sipe, 2006; Sipe et al., 2002), y diferentes manifestaciones clínicas en consumidores de cannabis (Haughey, Marshall, Schacht, Louis y Hutchison, 2008; Schacht, Selling y Hutchison, 2009).

El FAAH es el regulador crítico de los niveles endógenos de anandamida (Fezza, De Simone, Amadio y Maccarrone, 2008). El polimorfismo rs324420 del gen FAAH predice una sustitución de prolina en la posición 129 de la proteína por un residuo de treonina (P129T), lo que da como resultado una proteína que es más susceptible a la degradación proteolítica (Sipe, Chiang, Gerber, Beutler y Cravatt, 2002). Por tanto, el FAAH $385 A$ se asocia con una menor actividad enzimática. Los ratones knockout de FAAH han mostrado alterada la tolerancia y la dependencia de cannabis (Falenski et al., 2010), lo que sugiere que la actividad FAAH alterada puede modificar la señalización endocannabinoide en las áreas de control de recompensa y contribuir a la vulnerabilidad adictiva (Van Hell et al., 2012).

La literatura anterior sobre la relación entre el polimorfismo rs324420 y los patrones de uso de drogas parece ser extremadamente heterogénea y compleja (Hindocha et al., 2019; Melroy-Greif, Wilhelmsen y Ehlers, 2016; Tyndale et al., 2007). Se han identificado niveles más bajos de FAAH en sanos portadores A de rs324420 (Boileau et al., 2015). Se ha encontrado una reducida unión de FAAH en el cerebro en usuarios de cannabis en comparación con los controles. Además, una menor unión se ha asociado con la abstinencia, la impulsividad y el aumento de los niveles en sangre de cannabinoides (Boileau et al., 2016). En la muestra de usuarios de cannabis del estudio de Hindocha et al. (2019), los portadores A mostraron mayor sesgo hacia estímulos apetitivos, en comparación con los portadores CC. Hariri et al. (2009) encontraron que había una asociación en portadores de FAAH 385 a con posible aumento de la señalización endocannabinoide, y que había aumento de la reactividad del cuerpo estriado ventral relacionada con la 
recompensa y mayor impulsividad en comparación con los homocigotos C385. Por el contrario, Filbey, Schacht, Myers, Chavez y Hutchison (2010) identificaron mayor activación en las áreas de recompensa en portadores del alelo $\mathrm{C}$ en una muestra de consumidores habituales de marihuana.

Nuestros hallazgos han de interpretarse con precaución debido a la similitud de los niveles de significación para los modelos codominantes y de sobredominancia y por los reducidos números de la población de estudio (solo pocos casos presentaron el genotipo AA y los subgrupos de control de dependencia de cannabis eran pequeños).

\section{CNR2}

Diferentes estudios han demostrado que los receptores CB2 están presentes en las células progenitoras neurales, neuronas y células gliales. Además, la función del receptor CB2 no solo se ha relacionado con trastornos neurológicos que involucran neuroinflamación, sino también con trastornos neuropsiquiátricos como adicción a las drogas, psicosis, depresión y trastornos alimentarios (Onaivi et al., 2012).

No obstante, observamos una asociación entre los polimorfismos rs35761398 y rs12744386 en CNR2 y comorbilidad de esquizofrenia y dependencia de cannabis. Encontramos que el genotipo de alto funcionamiento de CNR2 se asoció con la esquizofrenia, pero solo en sujetos con dependencia de cannabis. Probamos varios modelos de herencia y descubrimos que la asociación estadística mejoraba cuando se asumía un modelo dominante para el alelo TT. Hasta donde sabemos, este es el primer estudio realizado en una población caucásica. La etnia debe tenerse en cuenta al interpretar nuestros resultados porque las distribuciones alélicas diferenciales se han descrito en la literatura científica previa sobre otros grupos étnicos. Por ejemplo, se ha informado una asociación entre la esquizofrenia y el haplotipo de función baja en una muestra de población japonesa (Ishiguro et al., 2010) y otros polimorfismos de CNR2 diferentes en muestras chinas (Tong et al., 2013), mientras que no se encontró asociación en muestras coreanas (Bae et al., 2014).

Banaszkiewicz, Biala y Kruk-Slomka (2020) realizaron una revisión sobre síntomas similares a la esquizofrenia inducidos a través de la modulación del receptor CB2 en modelos animales, lo que sugiere una función clave en la esquizofrenia. Se observaron conductas relacionadas con la esquizofrenia en ratones a los cuales se eliminaron los receptores CB2 (Ortega-Alvaro, Aracil-Fernández, García-Gutiérrez, Navarrete y Manzanares, 2011). Se sugiere que una falta de los receptores CB2 podría afectar el desarrollo neuronal, lo que induce alteraciones relevantes en varias áreas cerebrales, según los hallazgos que apoyan un papel proneurogénico del receptor CB2 en el control de procesos fundamentales de las células neuronales (Galve-Roperh, Aguado, Palazuelos y Guzman, 2008). Estos resultados parecen contrarios a nuestros datos, ya que re- lacionan la psicosis con una función más baja de los receptores CB2. Nuestros datos apoyarían una explicación alternativa: que la actividad excesiva de estos receptores podría facilitar este fenotipo psicótico. Por tanto, el consumo de cannabis podría interrumpir la diferenciación neuronal durante la adolescencia y provocar psicosis en sujetos vulnerables a través de un mecanismo que involucra CB2.

Por otro lado, los procesos inflamatorios e inmunológicos que interfieren con el desarrollo cerebral se discuten como una causa de esquizofrenia, y el receptor CB2 es un componente principal de estos procesos (Sahu et al., 2019). Las células gliales están implicadas en la patogénesis de la esquizofrenia y el receptor CB2 es relevante (De Almeida y Martins-de-Souza, 2018). Ha sido hipotetizado que un mayor número de células microgliales activadas en pacientes con esquizofrenia contribuyen a la patogénesis de la enfermedad (Juckel et al., 2011).

Además, en la enfermedad de Alzheimer, los receptores CB2 se expresan de forma abundante y selectiva en astrocitos y microglia asociados a la placa neurítica, respectivamente (Benito et al., 2003), y es probable que la activación de los receptores CB2 expresados por las células inmunitarias reduzca su respuesta antiviral, por tanto favoreciendo la entrada al SNC de monocitos infectados con el virus de inmunodeficiencia en simios (Benito et al., 2005).

Por tanto, la sobreactivación del receptor CB2 podría ser un factor de vulnerabilidad para la psicosis, y el consumo de cannabis podría provocar la psicosis en estos sujetos vulnerables. Se ha descubierto que el THC inhibe la respuesta quimiotáctica de la microglía mediante la activación del receptor CB2 (Cabral, Raborn, Griffin, Dennis y Marciano-Cabral, 2008). Además, el consumo de cannabis en el contexto de genotipos de receptores de cannabinoides específicos puede contribuir a anomalías de la sustancia blanca, lo que a su vez podría aumentar el riesgo de esquizofrenia (Ho, Wassink, Ziebell y Andreasen, 2011). Alteraciones de la sustancia blanca son relevantes en la esquizofrenia, y el consumo de cannabis durante la adolescencia tiene efectos específicos en estas anomalías (Peters, Blaas y de Haan, 2010).

Además, la expresión de las transcripciones del gen CNR2 en animales tratados con drogas de abuso aumenta en comparación con los controles (Ishiguro et al., 2007). Por tanto, el consumo de cannabis podría modificar la transcripción de CNR2 y, en sujetos con receptores CB2 altamente activados, podría contribuir a los síntomas psicóticos a través de un mecanismo desconocido. También se ha informado de que la remisión clínica de la esquizofrenia se acompaña de disminuciones significativas en los niveles de mRNA de CNR2 en las células mononucleares de sangre periférica (De Marchi et al., 2003).

Las limitaciones de este estudio están relacionadas con la naturaleza de los estudios de asociación y, por tanto, nuestros resultados deben interpretarse con cierta cautela. 
La fuerza de nuestros resultados está limitada por el pequeño tamaño muestral, particularmente en el grupo con dependencia de cannabis. Sería preferible replicar nuestros estudios genéticos en muestras independientes, ya que esto podría aclarar el posible papel de las variantes de los genes CNR1, CNR2 y FAAH. En segundo lugar, no puede descartarse la posibilidad de que un resultado ocurra por azar, a pesar de haber aplicado la corrección de Bonferroni. Por tanto, estos resultados deben confirmarse entre una población más grande. Asimismo, hubiera sido útil administrar la escala PANNS a los controles y en particular a los sujetos TCC, con el fin de descartar síntomas clínicos psicóticos. No se realizaron análisis de orina para detectar drogas en el grupo de control, por lo que es posible que algunos sujetos estuvieran consumiendo cannabis. Las pruebas de drogas se realizaron en el grupo de sujetos con esquizofrenia que no consumían cannabis.

A pesar de estas limitaciones, creemos que nuestro estudio ha identificado un importante factor protector genético frente a la psicosis asociada al cannabis en la población española, que merece mayor atención en futuras investigaciones. Esa investigación también debe examinar si las características fenotípicas específicas, como el perfil de los síntomas, la edad de inicio y la respuesta al tratamiento, están asociadas con el polimorfismo CNR2. No hay disponibles ningunos informes anteriores de estos polimorfismos en la psicosis asociada a los cannabinoides. La mutación detectada por este polimorfismo resulta en un cambio en la secuencia de aminoácidos de la proteína, por lo que se esperan consecuencias funcionales directas. Los sujetos con dependencia de cannabis con el genotipo TT exhibieron un riesgo significativamente mayor de psicosis. Estos hallazgos sugieren que la disfunción del sistema endocannabinoide debido a una mutación genética puede constituir un factor de riesgo para la psicosis asociada al cannabis.

Aparte de las variantes genéticas, también sería recomendable incluir variables epigenéticas en futuras investigaciones. Los cambios en la metilación del ADN en la región promotora de los genes $C N R 1$ en pacientes con esquizofrenia han sido descritos (D'Addario et al., 2017; Tao et al., 2020).

Por último, no encontramos una relación entre las variantes de $C N R 1$ y $F A A H$ y la psicosis. En general, nuestros hallazgos sugieren que las variantes de $F A A H$ se asocian con la dependencia de cannabis pero no con la esquizofrenia en pacientes españoles, lo que implicaría que las diferencias en la función endocannabinoide podrían tener un papel en la fisiopatología de esta enfermedad. La confirmación de nuestros hallazgos entre otras poblaciones y muestras independientes sería útil para el diseño de estrategias farmacológicas centradas en la profilaxis y el tratamiento de estos pacientes.

\section{Reconocimientos}

Los autores desean expresar su agradecimiento a: Margarita Agujetas, Dolores Baño, Angela Domenech, Rocío Molina, Enriqueta Ochoa, Ruth Olmos, Gerardo Pacheco, Estrella Salvador, José Luis Santos, Agustín Soto, Carmen Tosio y Diego Urgelés quienes ayudaron en la reclutación de los participantes del estudio, junto con Marta Marín y Tomasz Dukanovich que ayudaron con la traducción. El trabajo contó con el apoyo del Plan Nacional sobre Drogas Orden SAS/3031/2009, 26 de octubre.

\section{Conflicto de intereses}

Los autores declaran la inexistencia de conflicto de interés.

\section{Referencias}

Andreasen, N. (1995). Symptoms, signs, and diagnosis of schizophrenia. The Lancet,346, 477-481. doi:10.1016/ s0140-6736(95)91325-4.

Aas, M., Melle, I., Bettella, F., Djurovic, S., Le Hellard, S., Bjella, T.,... Tesli, M. (2017). Psychotic patients who used cannabis frequently before illness onset have higher genetic predisposition to schizophrenia than those who did not. Psychological Medicine, 48, 43-49. doi:10.1017/ S0033291717001209.

Bae, J. S., Kim, J. Y., Park, B. L., Kim, J. H., Kim, B., Park, C. S.,... Woo, S. (2014). Genetic association analysis of CNR1 and CNR2 polymorphisms with schizophrenia in a Korean population. Psychiatric Genetics, 24, 225-229. doi:10.1097/YPG.0000000000000047.

Ballon, N., Leroy, S., Roy, C., Bourdel, M. C., Charles-Nicolas, A., Krebs, M. O. y Poirier, M. F. (2006). (AAT) $\mathrm{n}$ repeat in the cannabinoid receptor gene (CNR1): Association with cocaine addiction in an African-Caribbean population. Pharmacogenomics Journal, 6, 126-130. doi:10.1038/sj.tpj.6500352.

Banaszkiewicz, I., Biala, G. y Kruk-Slomka, M. (2020). Contribution of CB2 receptors in schizophrenia-related symptoms in various animal models: Short review. Neuroscience E Biobehavioral Reviews, 114, 158-171. doi:10.1016/j.neubiorev.2020.04.020

Benito, C., Núñez, E., Tolón, R. M., Carrier, E. J., Rábano, A., Hillard, C. J. y Romero, J. (2003). Cannabinoid CB2 receptors and fatty acid amide hydrolase are selectively overexpressed in neuritic plaque-associated glia in $\mathrm{Al}-$ zheimer's disease brains. Journal of Neuroscience, 23, 111361114. doi:10.1523/JNEUROSCI.23-35-11136.2003.

Benito, C., Kim, W. K., Chavarría, I., Hillard, C. J., Mackie, K., Tolón, R. M.,... Romero, J. (2005). A glial endogenous cannabinoid system is upregulated in the brains of macaques with simian immunodeficiency virus-indu- 
ced encephalitis. Journal of Neuroscience, 23, 11136-11141. doi:10.1523/JNEUROSCI.3923-04.2005.

Bioque, M., Mas, S., Costanzo, M. C., Cabrera, B., Lobo, A., González-Pinto, A.,... Balanzá-Martínez, V. (2019). Gene-environment interaction between an endocannabinoid system genetic polymorphism and cannabis use in first episode of psychosis. European Neuropsychopharmacology, 29, 786-794. doi:10.1016/j.euroneuro.2019.04.005.

Boileau, I., Tyndale, R. F., Williams, B., Mansouri, E., Westwood, D. J., Le Foll, B.,... Tong, J. (2015). The fatty acid amide hydrolase C385A variant affects brain binding of the positron emission tomography tracer [11C] CURB. Journal of Cerebral Blood Flow and Metabolism, 35, 12371240. doi:10.1038/jcbfm.2015.119.

Boileau, I., Mansouri, E., Williams, B., Le Foll, B., Rusjan, P., Mizrahi, R.,.... Tong, J. (2016). Fatty acid amide hydrolase binding in brain of cannabis users: Imaging with the novel radiotracer [11C]CURB. Biological Psychiatry, 80, 691-701. doi:10.1016/j.biopsych.2016.04.012.

Borgan, F., Laurikainen, H., Veronese, M., Marques, T. R., Haaparanta-Solin, M., Solin, O.,... Howes, O. (2019). In vivo availability of cannabinoid 1 receptor levels in patients with first-episode psychosis. JAMA Psychiatry, 76, 1074. doi:10.1001/jamapsychiatry.2019.1427.

Cabral, G. A., Raborn, E. S., Griffin, L., Dennis, J. y Marciano-Cabral, F. (2008). CB 2 receptors in the brain: Role in central immune function. British Journal of Pharmacology, 153, 240-251. doi:10.1038/sj.bjp.0707584.

Chavarría-Siles, I., Contreras-Rojas, J., Hare, E., Walss-Bass, C., Quezada, P., Dassori, A.,... Escamilla, M. A. (2008). Cannabinoid receptor 1 gene (CNR1) and susceptibility to a quantitative phenotype for hebephrenic schizophrenia. American Journal of Medical Genetics, Part B: Neuropsychiatric Genetics, 147B, 279-284. doi:10.1002/ ajmg.b.30592.

Comings, D. E., Muhleman, D., Gade, R., Johnson, P., Verde, R., Saucier, G. y MacMurray, J. (1997). Cannabinoid receptor gene (CNR1): Association with IV drug use. Molecular Psychiatry, 2, 161-168. doi:10.1038/ sj.mp.4000247.

Covault, J., Gelernter, J. y Kranzler, H. (2001). Association study of cannabinoid receptor gene (CNR1) alleles and drug dependence. Molecular Psychiatry, 6, 501-502. doi:10.1038/sj.mp.4000925.

D’Addario, C., Micale, V., Di Bartolomeo, M., Stark, T., Pucci, M., Sulcova, A.,... Dell'Osso, B. (2017). A preliminary study of endocannabinoid system regulation in psychosis: Distinct alterations of CNR1 promoter DNA methylation in patients with schizophrenia. Schizophrenia Research, 188, 132-140. doi:10.1016/j.schres.2017.01.022.

Dawson, E. (1995). Identification of a highly polymorphic triplet repeat marker for the brain cannabinoid receptor gene: Use in linkage and association stu- dies of schizophrenia. Schizophrenia Research, 15, 37. doi:10.1016/0920-9964(95)95120-x.

De Almeida, V. y Martins-de-Souza, D. (2018). Cannabinoids and glial cells: Possible mechanism to understand schizophrenia. European Archives of Psychiatry and Clinical Neuroscience, 268, 727-737. doi:10.1007/s00406-0180874-6.

De Marchi, N., De Petrocellis, L., Orlando, P., Daniele, F., Fezza, F. y Di Marzo, V. (2003). Endocannabinoid signalling in the blood of patients with schizophrenia. Lipids in Health and Disease, 2, 5. doi:10.1186/1476-511X-2-1.

Deutsch, D. G., Ueda, N. y Yamamoto, S. (2002). The fatty acid amide hydrolase (FAAH). Prostaglandins Leukotrienes and Essential Fatty Acids, 66, 201-210. doi:10.1054/ plef.2001.0358.

Dong, M., Lu, L., Zhang, L., Zhang, Y. S., Ng, C. H., Ungvari, G. S.,... Xiang, Y. T. (2019). Quality of life in schizophrenia: A meta-analysis of comparative studies. Psychiatric Quarterly, 90, 519-532. doi:10.1007/s11126019-09633-4.

Fakhoury, M. (2017). Role of the endocannabinoid system in the pathophysiology of schizophrenia. Molecular Neurobiology, 54, 768-778. doi:10.1007/s12035-016-9697-5.

Falenski, K. W., Thorpe, A. J., Schlosburg, J. E., Cravatt, B. F., Abdullah, R. A., Smith, T. H.,... Sim-Selley, L. J. (2010). FAAH/Mice display differential tolerance, dependence, and cannabinoid receptor adaptation after $\delta$ 9-tetrahydrocannabinol and anandamide administration. Neuropsychopharmacology, 35, 1775-1787. doi:10.1038/npp.2010.44.

Fezza, F., De Simone, C., Amadio, D. y Maccarrone, M. (2008). Fatty acid amide hydrolase: A gate-keeper of the endocannabinoid system. Sub-Cellular Biochemistry, 49, 101-132. doi:10.1007/978-1-4020-8831-5_4.

Filbey, F. M., Schacht, J. P., Myers, U. S., Chavez, R. S. y Hutchison, K. E. (2010). Individual and additive effects of the CNR1 and FAAH genes on brain response to marijuana cues. Neuropsychopharmacology, 35, 967-975. doi:10.1038/npp.2009.200.

Flanagan, J. M., Gerber, A. L., Cadet, J. L., Beutler, E. y Sipe, J. C. (2006). The fatty acid amide hydrolase 385 A/A (P129T) variant: Haplotype analysis of an ancient missense mutation and validation of risk for drug addiction. Human Genetics, 120, 581-588. doi:10.1007/s00439006-0250-x.

Fonseca-Pedrero, E., Lucas-Molina, B., Pérez-Albéniz, A., Inchausti, F. y Ortuño-Sierra, J. (2019). Experiencias psicóticas atenuadas y consumo de cannabis en adolescentes de la población general.Adicciones, 32, 41. doi:10.20882/adicciones.1149.

French, L., Gray, C., Leonard, G., Perron, M., Pike, G. B., Richer, L.,... Paus, T. (2015). Early cannabis use, polygenic risk score for schizophrenia, and brain maturation 
in adolescence. JAMA Psychiatry, 72, 1002. doi:10.1001/ jamapsychiatry.2015.1131.

Galiègue, S., Mary, S., Marchand, J., Dussossoy, D., Carrière, D., Carayon, P.,... Casellas, P. (1995). Expression of central and peripheral cannabinoid receptors in human immune tissues and leukocyte subpopulations. European Journal of Biochemistry, 232, 54-61. doi:10.1111/j.1432-1033.1995.tb20780.x.

Galve-Roperh, I., Aguado, T., Palazuelos, J. y Guzman, M. (2008). Mechanisms of control of neuron survival by the endocannabinoid system. Current Pharmaceutical Design, 14, 2279-2288. doi:10.2174/138161208785740117.

García-Álvarez, L., Gomar, J., García-Portilla, M. y Bobes, J. (2019). Consumo de cannabis y alteraciones cognitivas en esquizofrenia y primeros episodios psicóticos. Adicciones, 31, 89-94. doi:10.20882/adicciones.1328.

García-Gutiérrez, M. S., Ortega-Álvaro, A., Busquets-García, A., Pérez-Ortiz, J. M., Caltana, L., Ricatti, M. J.,... Manzanares, J. (2013). Synaptic plasticity alterations associated with memory impairment induced by deletion of CB2 cannabinoid receptors. Neuropharmacology, 73, 388-396. doi:10.1016/j.neuropharm.2013.05.034.

Gerra, M. C., Jayanthi, S., Manfredini, M., Walther, D., Schroeder, J., Phillips, K. A.,... Donnini, C. (2018). Gene variants and educational attainment in cannabis use: Mediating role of DNA methylation. Translational Psychiatry, 8, 23. doi:10.1038/s41398-017-0087-1.

Gong, J. P., Onaivi, E. S., Ishiguro, H., Liu, Q. R., Tagliaferro, P. A., Brusco, A. y Uhl, G. R. (2006). Cannabinoid CB2 receptors: Immunohistochemical localization in rat brain. Brain Research, 1071, 10-23. doi:10.1016/j.brainres.2005.11.035.

Gouvêa, E. S., Santos, A. F., Ota, V. K., Mrad, V., Gadelha, A., Bressan, R. A.,... Belangero, S. I. (2017). The role of the CNR1 gene in schizophrenia: A systematic review including unpublished data. Revista Brasileira de Psiquiatria, 39, 160-171. doi:10.1590/1516-4446-2016-1969.

Hamdani, N., Tabeze, J. P., Ramoz, N., Ades, J., Hamon, M., Sarfati, Y.,... Gorwood, P. (2008). The CNR1 gene as a pharmacogenetic factor for antipsychotics rather than a susceptibility gene for schizophrenia. European Neuropsychopharmacology, 18, 34-40. doi:10.1016/j.euroneuro.2007.05.005.

Hariri, A. R., Gorka, A., Hyde, L. W., Kimak, M., Halder, I., Ducci, F.,... Manuck, S. B. (2009). Divergent effects of genetic variation in endocannabinoid signaling on human threat- and reward-related brain function. Biological Psychiatry, 66, 9-16. doi:10.1016/j.biopsych.2008.10.047.

Hartman, C. A., Hopfer, C. J., Haberstick, B., Rhee, S. H., Crowley, T. J., Corley, R. P.,... Ehringer, M. A. (2009). The association between cannabinoid receptor 1 gene (CNR1) and cannabis dependence symptoms in adolescents and young adults. Drug and Alcohol Dependence, 104, 11-16. doi:10.1016/j.drugalcdep.2009.01.022.
Haughey, H. M., Marshall, E., Schacht, J. P., Louis, A. y Hutchison, K. E. (2008). Marijuana withdrawal and craving: Influence of the cannabinoid receptor 1 (CNR1) and fatty acid amide hydrolase (FAAH) genes. Addiction, 103, 1678-1686. doi:10.1111/j.1360-0443.2008.02292.x.

Heller, D., Schneider, U., Seifert, J., Cimander, K. F. y Stuhrmann, M. (2001). The cannabinoid receptor gene (CNR1) is not affected in German i.v. drug users. Addiction Biology, 6, 183-187. doi:10.1080/13556210020040271.

Herkenham, M., Lynn, A. B., Johnson, M. R., Melvin, L. S., De Costa, B. R. y Rice, K. C. (1991). Characterization and localization of cannabinoid receptors in rat brain: A quantitative in vitro autoradiographic study. Journal of Neuroscience, 11, 563-583. doi:10.1523/jneurosci.11-02-00563.1991.

Hindocha, C., Freeman, T., Bloomfield, M., Bramon, E., Morgan, C. y Curran, H. V. (2019). P.147 Acute effects of cannabinoids on addiction endophenotypes are moderated by genes encoding the cannabinoid receptor 1 and FAAH enzyme. European Neuropsychopharmacology, 29, S116-S117. doi:10.1016/j.euroneuro.2019.09.196.

Hindocha, C., Quattrone, D., Freeman, T. P., Murray, R. M., Mondelli, V., Breen, G.,... Di Forti, M. (2020). Do AKT1, COMT and FAAH influence reports of acute cannabis intoxication experiences in patients with first episode psychosis, controls and young adult cannabis users? Translational Psychiatry, 10, 143. doi:10.1038/ s41398-020-0823-9.

Ho, B. C., Wassink, T. H., Ziebell, S. y Andreasen, N. C. (2011). Cannabinoid receptor 1 gene polymorphisms and marijuana misuse interactions on white matter and cognitive deficits in schizophrenia. Schizophrenia Research, 128, 66-75. doi:10.1016/j.schres.2011.02.021.

Ishiguro, H., Iwasaki, S., Teasenfitz, L., Higuchi, S., Horiuchi, Y., Saito, T.,... Onaivi, E. S. (2007). Involvement of cannabinoid CB2 receptor in alcohol preference in mice and alcoholism in humans. Pharmacogenomics Journal, 7, 380-385. doi:10.1038/sj.tpj.6500431.

Ishiguro, H., Horiuchi, Y., Ishikawa, M., Koga, M., Imai, K., Suzuki, Y.,... Arinami, T. (2010). Brain cannabinoid CB2 receptor in schizophrenia. Biological Psychiatry, 67, 974982. doi:10.1016/j.biopsych.2009.09.024.

Javed, H., Azimullah, S., Haque, M. E. y Ojha, S. K. (2016). Cannabinoid type 2 (CB2) receptors activation protects against oxidative stress and neuroinflammation associated dopaminergic neurodegeneration in rotenone model of parkinson's disease. Frontiers in Neuroscience, 10, 321. doi:10.3389/fnins.2016.00321.

Juckel, G., Manitz, M. P., Brüne, M., Friebe, A., Heneka, M. T. y Wolf, R. J. (2011). Microglial activation in a neuroinflammational animal model of schizophrenia - a pilot study. Schizophrenia Research, 131, 96-100. doi:10.1016/j. schres.2011.06.018. 
Kay, S. R., Fiszbein, A. y Opler, L. A. (1987). The positive and negative syndrome scale (PANSS) for schizophrenia. Schizophrenia Bulletin, 13, 261-276.

Kong, W., Li, H., Tuma, R. F. y Ganea, D. (2014). Selective CB2 receptor activation ameliorates EAE by reducing Th17 differentiation and immune cell accumulation in the CNS. Cellular Immunology, 287, 1-17. doi:10.1016/j. cellimm.2013.11.002.

Krebs, M. O., Morvan, Y., Jay, T., Gaillard, R. y Kebir, O. (2014). Psychotomimetic effects at initiation of cannabis use are associated with cannabinoid receptor 1 (CNR1) variants in healthy students. Molecular Psychiatry, 19, 402403. doi:10.1038/mp.2013.188.

Leroy, S., Griffon, N., Bourdel, M. C., Olié, J. P., Poirier, M. F. y Krebs, M. O. (2001). Schizophrenia and the cannabinoid receptor type 1 (CB1): Association study using a single-base polymorphism in coding exon 1. American Journal of Medical Genetics - Neuropsychiatric Genetics, 105, 749-752. doi:10.1002/ajmg.10038.

Li, T., Liu, X., Zhu, Z. H., Zhao, J., Hu, X., Ball, D. M.,.... Collier, D. A. (2000). No association between (AAT)n repeats in the cannabinoid receptor gene (CNR1) and heroin abuse in a Chinese population. Molecular Psychiatry, 5, 128-130. doi:10.1038/sj.mp.4000670.

Malfitano, A. M., Basu, S., Maresz, K., Bifulco, M. y Dittel, B. N. (2014). What we know and do not know about the cannabinoid receptor 2 (CB2). Seminars in Immunology, 26, 369-379. doi:10.1016/j.smim.2014.04.002.

Manzanares, J., Cabañero, D., Puente, N., García-Gutiérrez, M. S., Grandes, P. y Maldonado, R. (2018). Role of the endocannabinoid system in drug addiction. Biochemical Pharmacology, 157, 108-121. doi:10.1016/j. bcp.2018.09.013.

Marconi, A., Di Forti, M., Lewis, C. M., Murray, R. M. y Vassos, E. (2016). Meta-Analysis of the association between the level of cannabis use and risk of psychosis. Schizophrenia Bulletin, 42, 1262-1269. doi:10.1093/schbul/sbw003.

Martínez-Gras, I., Hoenicka, J., Ponce, G., Rodríguez-Jiménez, R., Jiménez-Arriero, M. A., Pérez-Hernandez, E., ... Rubio, G. (2006). (AAT)n repeat in the cannabinoid receptor gene, CNR1: Association with schizophrenia in a Spanish population. European Archives of Psychiatry and Clinical Neuroscience, 256, 437-441. doi:10.1007/s00406006-0665-3.

Melroy-Greif, W. E., Wilhelmsen, K. C. y Ehlers, C. L. (2016). Genetic variation in FAAH is associated with cannabis use disorders in a young adult sample of Mexican Americans. Drug and Alcohol Dependence, 166, 249253. doi:10.1016/j.drugalcdep.2016.06.021.

Minichino, A., Senior, M., Brondino, N., Zhang, S. H., Godwlewska, B. R., Burnet, P. W. J.,... Lennox, B. R. (2019). Measuring disturbance of the endocannabinoid system in psychosis: A systematic review and meta-analysis.
JAMA Psychiatry, 76, 914-923. doi:10.1001/jamapsychiatry.2019.0970.

Morita, Y., Ujike, H., Tanaka, Y., Uchida, N., Nomura, A., Ohtani, K.,... Kuroda, S. (2005). A nonsynonymous polymorphism in the human fatty acid amide hydrolase gene did not associate with either methamphetamine dependence or schizophrenia. Neuroscience Letters, 376, 182-187. doi:10.1016/j.neulet.2004.11.050.

Onaivi, E. S., Ishiguro, H., Gong, J. P., Patel, S., Perchuk, A., Meozzi, P. A.,... Uhl, G. R. (2006). Discovery of the presence and functional expression of cannabinoid CB2 receptors in brain. Annals of the New York Academy of Sciences, 1074, 514-536. doi:10.1196/annals.1369.052.

Onaivi, E. S., Ishiguro, H., Gu, S. y Liu, Q. R. (2012). CNS effects of CB2 cannabinoid receptors: Beyond neuro-immuno-cannabinoid activity. Journal of Psychopharmacology, 26, 92-103. doi:10.1177/0269881111400652.

Ortega-Alvaro, A., Aracil-Fernández, A., García-Gutiérrez, M. S., Navarrete, F. y Manzanares, J. (2011). Deletion of CB2 cannabinoid receptor induces schizophrenia-related behaviors in mice. Neuropsychopharmacology, 36, 14891504. doi:10.1038/npp.2011.34.

Patel, M. M., Nielsen, D. A., Kosten, T. R., De La Garza, R., Newton, T. F. y Verrico, C. D. (2018). FAAH variant Pro129Thr modulates subjective effects produced by cocaine administration. American Journal on Addictions, 27, 567-573. doi: 10.1111/ajad.12788.

Peralta, V. y Cuesta, M. J. (1994). Psychometric properties of the positive and negative syndrome scale (PANSS) in schizophrenia. Psychiatry Research, 53, 31-40.

Peters, B. D., Blaas, J. y de Haan, L. (2010). Diffusion tensor imaging in the early phase of schizophrenia: What have we learned? Journal of Psychiatric Research, 44, 9931004. doi:10.1016/j.jpsychires.2010.05.003.

Parkar, S. R., Ramanathan, S., Nair, N., Batra, S. A., Adarkar, S. A., Kund, P.,... Moghe, S. H. (2011). Are the effects of cannabis dependence on glucose metabolism similar to schizophrenia? An FDG PET understanding. Indian Journal of Psychiatry, 53, 13-20. doi:10.4103/00195545.75552.

Rodríguez-Muñoz, M., Sánchez-Blázquez, P., Callado, L. F., Meana, J. J. y Garzón-Niño, J. (2017). Schizophrenia and depression, two poles of endocannabinoid system deregulation. Translational Psychiatry, 7, 1291. doi:10.1038/ s41398-017-0029-y.

Rojnic, M., Bosnjak, D., Ganoci, L., Makaric, P., Kekin, I., Rossini, L.,... Bozina, N. (2019). Association of CNR1 genotypes with changes in neurocognitive performance after eighteen-month treatment in patients with first-episode psychosis. European Psychiatry, 61, 88-96. doi:10.1016/j.eurpsy.2019.07.004.

Sahu, P., Mudgal, J., Arora, D., Kinra, M., Mallik, S. B., Rao, C. M.,... Nampoothiri, M. (2019). Cannabinoid 
receptor 2 activation mitigates lipopolysaccharide-induced neuroinflammation and sickness behavior in mice. Psychopharmacology, 236, 1829-1838. doi:10.1007/s00213019-5166-y.

Schacht, J. P., Selling, R. E. y Hutchison, K. E. (2009). Intermediate cannabis dependence phenotypes and the FAAH C385A variant: An exploratory analysis. Psychopharmacology, 203, 511-517. doi:10.1007/s00213-008-1397-z.

Seifert, J., Ossege, S., Emrich, H. M., Schneider, U. y Stuhrmann, M. (2007). No association of CNR1 gene variations with susceptibility to schizophrenia. Neuroscience Letters, 426, 29-33. doi:10.1016/j.neulet.2007.08.008.

Sipe, J. C., Chiang, K., Gerber, A. L., Beutler, E. y Cravatt, B. F. (2002). A missense mutation in human fatty acid amide hydrolase associated with problem drug use. Proceedings of the National Academy of Sciences of the United States of America, 99, 8394-8399. doi:10.1073/pnas.082235799.

Tao, R., Li, C., Jaffe, A., Shin, J., Deep-Soboslay, A., Yamin, R.,.... Hyde, T.M. (2020). Cannabinoid receptor CNR1 expression and DNA methylation in human prefrontal cortex, hippocampus and caudate in brain development and schizophrenia. Translational Psychiatry, 10, 158. doi:10.1038/s41398-020-0832-8.

Tong, D., He, S., Wang, L., Jin, L., Si, P. y Cheng, X. (2013). Association of single-nucleotide polymorphisms in the cannabinoid receptor 2 gene with schizophrenia in the Han Chinese population. Journal of Molecular Neuroscience, 51, 454-460. doi:10.1007/s12031-013-0062-0.

Tsai, S. J., Wang, Y. C. y Hong, C. J. (2000). Association study of a cannabinoid receptor gene (CNR1) polymorphism and schizophrenia. Psychiatric Genetics, 10, 149151. doi:10.1097/00041444-200010030-00008.

Tyndale, R. F., Payne, J. I., Gerber, A. L. y Sipe, J. C. (2007). The fatty acid amide hydrolase C385A (P129T) missen- se variant in cannabis users: Studies of drug use and dependence in caucasians. American Journal of Medical Genetics, Part B: Neuropsychiatric Genetics, 144B, 660-666. doi:10.1002/ajmg.b.30491.

Ujike, H., Takaki, M., Nakata, K., Tanaka, Y., Takeda, T., Kodama, M.,... Kuroda, S. (2002). CNR1, central cannabinoid receptor gene, associated with susceptibility to hebephrenic schizophrenia. Molecular Psychiatry, 7, 515518. doi:10.1038/sj.mp.4001029.

Xi, Z. X., Peng, X. Q., Li, X., Song, R., Zhang, H. Y., Liu, Q. R.,.. Gardner, E. L. (2011). Brain cannabinoid CB2 receptors modulate cocaine's actions in mice. Nature Neuroscience, 14, 1160-1166. doi:10.1038/nn.2874.

Van Hell, H. H., Jager, G., Bossong, M. G., Brouwer, A., Jansma, J. M., Zuurman, L.,... Ramsey, N. F. (2012). Involvement of the endocannabinoid system in reward processing in the human brain. Psychopharmacology, 219, 981-990. doi:10.1007/s00213-011-2428-8.

Watts, J. J., Jacobson, M. R., Lalang, N., Boileau, I., Tyndale, R. F., Kiang, M.,... Mizrahi, R. (2020). Imaging brain fatty acid amide hydrolase in untreated patients with psychosis. Biological Psychiatry, 88, 727-735. doi:10.1016/j. biopsych.2020.03.003.

Zammit, S., Spurlock, G., Williams, H., Norton, N., Williams, N., O’Donovan, M. C.,... Owen, M. J. (2007). Genotype effects of CHRNA7, CNRI and COMT in schizophrenia: Interactions with tobacco and cannabis use. British Journal of Psychiatry, 191, 402-407. doi:10.1192/ bjp.bp.107.036129.

Zhang, W., Liu, H., Deng, X., Ma, Y. y Liu, Y. (2020). FAAH levels and its genetic polymorphism association with susceptibility to methamphetamine dependence. Annals of Human Genetics, 84, 259-270. doi:10.1111/ahg.12368. 
